\title{
$\checkmark$ EDITOR'S CHOICE \\ Prognostic significance of frequent premature ventricular contractions originating from the ventricular outflow tract in patients with normal left ventricular function
}

\author{
S Niwano, Y Wakisaka, H Niwano, H Fukaya, S Kurokawa, M Kiryu, Y Hatakeyama, \\ T Izumi
}

\section{See Editorial, p 1209}

Department of Cardio-angiology, Kitasato University School of Medicine, Sagamihara City, Kanagawa, Japan

\section{Correspondence to:} Professor S Niwano, Department of Cardio-angiology, Kitasato University School of Medicine,

1-15-1 Kitasato, Sagamihara City, Kanagawa, Japan 2288555;

shniwano@med.kitasato-u.ac.jp

Accepted 23 December 2008 Published Online First 7 May 2009
ABSTRACT

Background: Recently, it has been reported that frequent premature ventricular contractions (PVCs) may be associated with causing heart failure in patients with left ventricular (LV) dysfunction. However, the prognostic significance of frequent PVCs in asymptomatic patients with a normal LV function is unclear.

Methods: Two hundred and thirty-nine consecutive patients presenting with frequent PVCs ( $>1000$ beats/ day) originating from the right or left ventricular outflow tract without any detectable heart disease were enrolled in the study. Structural heart disease was ruled out by echocardiography and cardiac magnetic resonance imaging, and Holter-ECG monitoring was repeated two or three times to evaluate the PVC prevalence at the initial evaluation. All patients were followed up for at least 4 years, and further observation was continued if possible.

Results: During an observation period of 5.6 (1.7) years, no patients exhibited any serious cardiac events. Although there was no significant change in the mean LV ejection fraction (LVEF) and mean LV diastolic dimension (LVDd), there was a significant negative correlation between the PVC prevalence and $\Delta$ LVEF $(p<0.001)$ and positive correlation between the PVC prevalence and $\triangle \mathrm{LVDd}$ $(p<0.001)$. When the development of LV dysfunction was defined as $\Delta \mathrm{LVEF}>-6 \%, 13$ patients exhibited LV dysfunction. For the prediction of the development of LV dysfunction, PVC prevalence and LVEF at the initial evaluation were independent predicting factors $(p<0.01)$. Conclusion: Although the prognosis in patients with frequent PVCs was considered relatively benign, attention should be paid to the progression of the LV dysfunction during a long-term observation, especially in patients with a high PVC prevalence.

It is relatively common for cardiovascular clinicians to see patients with frequent premature ventricular contractions (PVCs) originating from the right or left ventricular outflow tract. Because these PVCs may appear in patients without any overt cardiovascular disease, the pathogenesis of that arrhythmia would be considered idiopathic. Although some of those patients may have sustained ventricular tachycardia (VT) and require catheter ablation for preventative therapy, most of the remaining patients exhibit no severe clinical symptoms, and so the prognosis in those patients with frequent PVCs but without any overt symptoms, is believed to be relatively benign. ${ }^{1}$
Recently, it has been reported that frequent PVCs might contribute to worsening the ventricular function in patients with slight left ventricular (LV) dysfunction, ${ }^{2-5}$ and the elimination of the PVCs by catheter ablation would improve the ventricular function during the clinical follow-up. ${ }^{6-}$ ${ }^{10}$ However, the prognostic significance of frequent PVCs in asymptomatic or less symptomatic patients with a normal LV function is unclear. In the present study, a long-term prospective cohort observation was performed in patients with frequent PVCs and a normal LV function in order to clarify the prognosis in such patients.

\section{METHODS}

\section{Patients and initial evaluation}

The study population consisted of 281 consecutive patients with frequent PVCs ( $>1000$ beats/day on Holter monitoring, Model 563 StrataScan, Del Mar Avionics, Boston, Massachusetts) originating from the right or left ventricular outflow tract without any detectable heart disease. Most of the patients were asymptomatic and assigned to further examination due to the recording of frequent PVCs in the 12-lead ECG in a routine health check. The outflow tract PVCs were diagnosed by a ORS configuration in the 12-lead body surface ECG consisting of tall $\mathrm{R}$ waves in the inferior leads and a complete left bundle branch block pattern in the chest leads (fig 1). However, 42/281 patients who exhibited syncope or faintness due to non-sustained VT or slight LV dysfunction (LV ejection fraction (LVEF) $<55 \%$ on the echocardiogram) were assigned to catheter ablation therapy and excluded from further observation. Out of the remaining 239 patients, 121 were female and 118 male, and the mean age was 43 (SD 13) years (table 1). Structural heart diseases including arrhythmogenic right ventricular cardiomyopathy were denied by echocardiography in all cases, and MRI in selected cases (63/239) who agreed to take the examination. The LVEF and left ventricular diastolic dimension (LVDd) were evaluated from the echocardiogram. All echocardiographical recordings and measurements were performed by three observers who were blinded to the clinical information. All measurements were made from the recordings during continuation of sinus rhythm, and the LVEF was calculated by the standard area-length method. The inter- and intraobserver variabilities of the measurements 
were evaluated in the pilot measurement in 10 healthy volunteers and were $<3 \%$ for the LVEF (ie, $<1.8 \%$ ) and $<2 \%$ for the LVDd (ie, $<1 \mathrm{~mm}$ ). Because all patients were basically asymptomatic and exhibited no evidence of structural heart disease, invasive evaluations such as cardiac catheterisation, coronary angiography or myocardial biopsy were not indicated. To evaluate the PVC prevalence in each patient, Holter ECG recordings were repeated at least two or three times to average the day-to-day variation at the initial evaluation. From those Holter recordings, the mean PVC prevalence (beats/day) and presence of non-sustained VT ( $>3$ beats) were evaluated. In the patients who complained of chest discomfort due to the PVCs, beta-blockers (atenolol 25-50 mg/day or bisoprolol $2.5-5 \mathrm{mg}$ / day) were allowed to be prescribed to control the PVC number (table 1), but in such cases, the LVEF and PVC prevalence were re-evaluated under the stable use of the beta-blockers, and the subsequent follow-up observations were also performed under that same prescription.

\section{Observation protocol}

The 239 patients who underwent the initial evaluation were enrolled for the long-term prospective observation. All patients were followed up for at least 4 years under the same therapeutic conditions, and further observation was continued when possible (achieved in 189/239 patients). The echocardiography and Holter ECG recordings (two or three times) were repeated once every year, and the LVEF and PVC prevalence determined from the Holter ECG recordings was evaluated. During the observation period, the incidence of clinical events such as sudden death, and the appearance of new-onset sustained VT, syncope or heart failure was evaluated. The changes in the LVEF and LVDd at the 4-year and latest time points were evaluated by comparing them with that at the initial evaluation. To evaluate the relationship between the change in the LVEF or LVDd and the PVC prevalence, the patients were divided into three groups according to their PVC prevalence, that is, highly frequent: PVCs $>20000$ beats/day, moderately frequent: PVCs between 5000 and 20000 beats/day, and less frequent: PVCs between 1000 and 5000 beats/day, and the LVEF and clinical data were compared among those three groups. Additionally, to evaluate the predicting factor for the development of LV dysfunction, the patients were divided into two groups with and without LV dysfunction, and clinical parameters were compared between the two groups. All studies were performed under the approval of the Clinical Studies and Ethics Committee of Kitasato University Hospital.

\section{Statistics}

All values are expressed as the mean (SD). The statistical analyses were performed with a one-way ANOVA test. A p value of $<0.05$ was considered significant.

\section{RESULTS}

\section{Prognosis and clinical characteristics of the patients}

The basic 4-year follow-up was completed in all patients and further observation was continued in 189/239 patients for another 1-4 years, resulting in a "latest" evaluation time point of 5.6 (1.7) years in total. During the observational period, no patients exhibited any clinical events, such as sudden death, new-onset VT, syncope or heart failure with any overt LV dysfunction.

Table 1 shows the clinical characteristics of the total patients and subgroups divided according to their PVC prevalence. As a result, 46 patients were classified into the group with highly frequent PVCs, 105 into the group with moderately frequent PVCs and 88 into the group with less frequent PVCs. There was no significant difference in the basic clinical characteristics among the three groups except for the PVC prevalence, day-today variability of PVCs and presence of non-sustained VT.

\section{LVEF, LVDd and PVC prevalence}

There were no significant changes in either of the LVEF, LVDd or PVC prevalence exhibited in any of the patients over the time course (table 1). However, a small population of patients exhibited a decrease in the LVEF and an increase in LVDd, but no patients suffered from any overt heart failure. Figure 2 shows the relationship between the PVC prevalence and change in the LVEF during the observation period, that is, $\triangle \mathrm{LVEF}=$ (LVEF at the evaluation time point)-(initial LVEF). The left panel shows the data after the basic 4-year follow-up, and the right panel the data at the latest follow-up visit. Although the change in the
Figure 1 Typical configuration of outflow tract premature ventricular contraction (PVCs) in the 12-lead ECG. This figure shows a representative example of the QRS configuration of PVCs originating in the outflow tract. The PVCs exhibit a tall $R$ wave in the inferior leads and a complete left bundle branch block pattern. See the text for details.

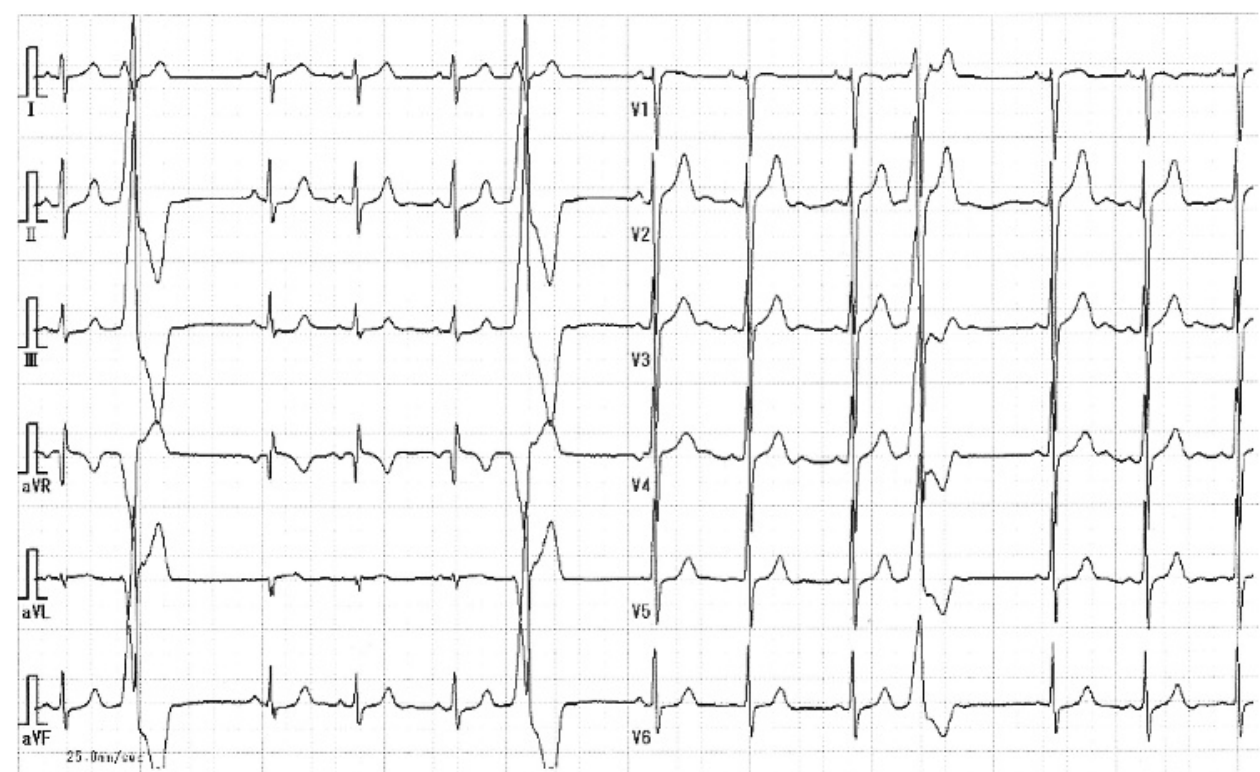


Table 1 Clinical characteristics of the patient groups

\begin{tabular}{|c|c|c|c|c|c|}
\hline & Total & Highly & Moderately & Less & p Value \\
\hline No & 239 & 46 & 105 & 88 & \\
\hline Age (years) & $43(13)$ & $44(15)$ & $43(12)$ & $42(12)$ & 0.336 \\
\hline Gender (female:male) & $121: 118$ & $23: 23$ & $53: 52$ & $45: 43$ & 0.991 \\
\hline $\begin{array}{l}\text { Systolic blood pressure } \\
(\mathrm{mm} \mathrm{Hg})\end{array}$ & $132(11)$ & $130(12)$ & $133(11)$ & $132(11)$ & 0.212 \\
\hline $\begin{array}{l}\text { Diastolic blood pressure } \\
(\mathrm{mm} \mathrm{Hg})\end{array}$ & $76(7)$ & $77(8)$ & $77(7)$ & $75(7)$ & 0.279 \\
\hline \multirow[t]{2}{*}{ Complications } & HT 16, HL 11 & HT 2, HL 3 & HT 7, HL 3 & HT 7, HL 3 & 0.776 \\
\hline & DM 9 & DM 1 & DM 5 & DM 3 & \\
\hline Beta-blockers (n) & 21 & 2 & 9 & 10 & 0.393 \\
\hline \multicolumn{6}{|l|}{$\begin{array}{l}\text { Left ventricular ejection } \\
\text { fraction }(\%)\end{array}$} \\
\hline Initial & $64(7)$ & $64(7)$ & $64(7)$ & $64(6)$ & 0.863 \\
\hline 4-year & $63(6)$ & $62(6)^{*}$ & $64(6)$ & $64(6)$ & 0.132 \\
\hline Latest & $63(6)$ & $61(6)^{*}$ & $63(6)^{*}$ & $64(6)$ & $0.011 \dagger$ \\
\hline \multicolumn{6}{|l|}{$\begin{array}{l}\text { Left ventricular diastolic } \\
\text { dimension }(\mathrm{mm})\end{array}$} \\
\hline Initial & $46(4)$ & $46(4)$ & $45(4)$ & $45(4)$ & 0.206 \\
\hline 4-year & $46(4)$ & $48(3)$ & $46(4)$ & $45(4)$ & $0.004 \dagger$ \\
\hline Latest & $46(4)$ & $48(3)$ & $46(4)$ & $46(4)$ & $0.001 \dagger$ \\
\hline \multicolumn{6}{|l|}{ PVCs (beats/day) } \\
\hline Initial & 12289 (12 238) & 33197 (10 164) & 11474 (4296) & $2323(1436)$ & $<0.001 \dagger$ \\
\hline 4-year & 12786 (11 880) & 30856 (12 310) & $12645(6003)$ & $3510(2214)$ & $<0.001 \dagger$ \\
\hline Latest & 13568 (12 961) & 32499 (14 219) & $13698(6961)$ & $3516(2317)$ & $<0.001 \dagger$ \\
\hline \multicolumn{6}{|l|}{$\begin{array}{l}\text { Day-to-day variability of } \\
\text { PVCs (beats/day) }\end{array}$} \\
\hline Initial & $2420(2935)$ & 6998 (3057) & 2197 (1578) & $296(234)$ & $<0.001 \uparrow$ \\
\hline 4-year & 2672 (2868) & $6582(3234)$ & 2246 (1428) & 372 (282) & $<0.001 \dagger$ \\
\hline Latest & $2486(2685)$ & 7029 (3167) & $2145(1522)$ & $321(256)$ & $<0.001 \uparrow$ \\
\hline \multicolumn{6}{|l|}{ NSVT (>3 beats, $\%$ ) } \\
\hline Initial & 18.8 & 34.8 & 15.2 & 14.8 & $0.008 \dagger$ \\
\hline 4-year & 15.1 & 39.1 & 11.4 & 6.8 & $<0.001 \dagger$ \\
\hline Latest & 20.1 & 39.1 & 16.1 & 14.8 & $0.016 \dagger$ \\
\hline
\end{tabular}

Initial, 4-year and latest indicate the evaluation time points.

${ }^{*} p<0.05$ versus the data during the initial evaluation.

$\uparrow$ Statistically significant differences among the three groups.

DM, diabetis mellitus; HL, hyperlipidaemia; HT, hypertension; NSVT, presence of non-sustained ventricular tachycardia; PVC, premature ventricular contraction.

LVEF in most of the patients remained within $\pm 6 \%$ ( $=$ standard deviation of the mean LVEF), several patients exhibited a relatively large decrease in the LVEF, and there was a weak but significant negative correlation between the PVC prevalence and $\triangle L V E F$ for both the 4-year and 5.6 (1.7)-year observation periods. Figure 3 shows the relationship between the PVC prevalence and change in the LVDd during the observation period, that is, $\triangle \mathrm{LVDd}=(\mathrm{LVDd}$ at the evaluation time point)-(initial LVDd). Similar to fig 2, the left panel shows the data after the 4-year follow-up, and the right panel the data at the latest follow-up. Although most of the patients exhibited only small changes in the LVDd measurement, several patients exhibited a relatively large increase in the LVDd, and there was a weak but significant positive correlation between the PVC prevalence and $\triangle$ LVDd for both the 4-year and 5.6 (1.7)-year observation periods. At 4 years and the latest time point, $\triangle \mathrm{LVEF}$ and $\triangle \mathrm{LVDd}$ exhibited a strong correlation, so these changes were considered to appear proportionally (fig 4).

Figures 5, 6 and table 1 show a comparison of the data among the three groups divided according to the PVC prevalence at the initial evaluation. Because these subgroups were classified based on the PVC prevalence, the total number of PVC differeds significantly, and the day-to-day variability of the number of PVCs and presence of non-sustained VT also exhibited a significant difference among the three groups (table 1). However, the PVC prevalence, day-to-day variability of the PVCs and presence of non-sustained VT did not exhibit any significant changes over the time course in any of the groups, indicating that the PVC prevalence detected in the Holter-ECG recordings seemed to be relatively reliable when it was calculated from 2-3 Holter recordings in order to exclude any day-to-day variation. Figure 5 shows the change in the LVEF and the LVDd over the time course in each subgroup. Although the less frequent PVC group did not exhibit any significant changes in the LVEF or LVDd, the highly frequent PVC group exhibited a significant decrease in the LVEF and an increase in the LVDd at the follow-ups at 4 years and later. The moderately frequent PVC group did not exhibit any significant decrease in the LVEF at the 4-year time point, but there was a significant decrease in the LVEF at the latest follow-up point. The LVDd did not exhibit any significant change in this subgroup. There was a significant difference in the mean LVEF between the less and highly frequent PVC groups at the latest follow-up. In contrast, the mean LVDd was significantly larger in the highly frequent PVC group than in the less frequent group at both 4 years and the latest follow-up time points. Figure 6 shows a comparison of the $\triangle \mathrm{LVEF}$ and $\triangle \mathrm{LVDd}$ among the three groups. At the 4-year and latest observation points, the decrease in the 
Figure 2 Relationship between the premature ventricular contraction (PVC) prevalence and change in left ventricular ejection fraction ( $\triangle \mathrm{LVEF}$ ) during the 4year or 5.6 (SD 1.7)-year observational periods. The $\triangle \mathrm{LVEF}$ was calculated as (LVEF at the evaluation time point)-(initial LVEF). The left panel shows the data after the basic 4-year follow-up, and the right panel the data at the latest follow-up. Although the LVEF in most patients remained within a change of $\pm 6 \%$, several patients exhibited a relatively large decrease in the LVEF, and there was a weak but significant negative correlation between the PVC prevalence and $\triangle \mathrm{LVEF}$ at the 4-year and 5.6 (1.7)year follow-ups. See text for details.

4 years

$\triangle \operatorname{LVEF}(\%)$

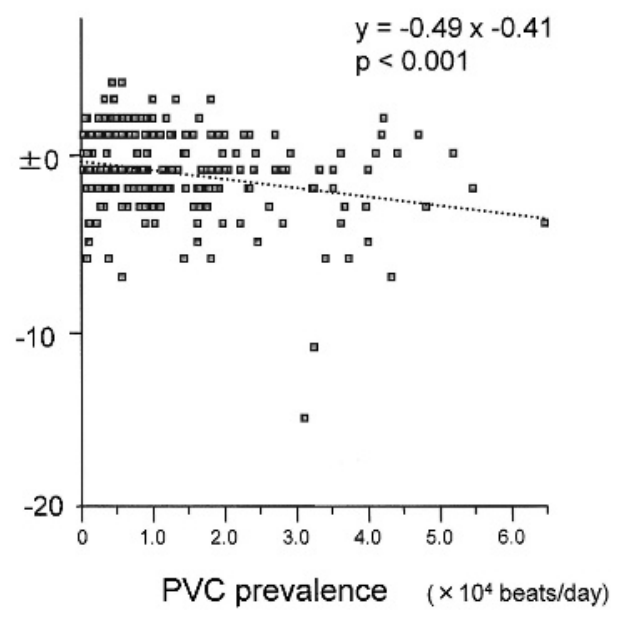

Latest $(5.6 \pm 1.7$ years $)$

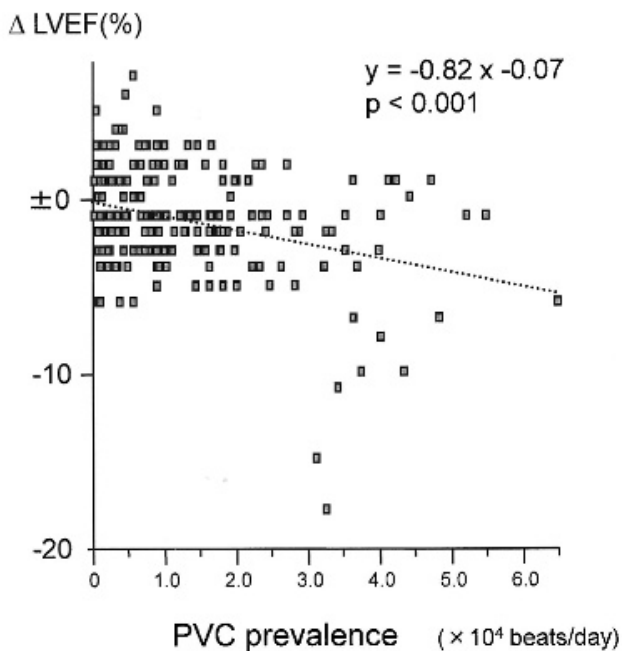

LVEF, that is, negative $\triangle L V E F$, and increase in the LVDd, that is, positive $\triangle \mathrm{LVDd}$, were larger in the highly frequent PVC group than in the less or moderately frequent PVC groups.

To clarify whether or not the observation period was a determining factor for a larger decrease in the LVEF or increase in the LVDd, the relationship between the observation period and $\triangle L V E F$ and $\triangle L V D d$ at the latest follow-up was evaluated (fig 7). Interestingly, in the patients who exhibited a larger decrease in the LVEF or a larger increase in the LVDd, the change in the $\triangle$ LVEF or $\triangle$ LVDd appeared within a relatively earlier period, that is, 4-8 years. As a result, there was no significant correlation between the observation period and $\triangle \mathrm{LVEF}$ or $\triangle \mathrm{LVDd}$, indicating that the observation period was not a determinant factor for the decrease in the LVEF or the increase in the LVDd.

\section{Prediction of the development of LV dysfunction}

To evaluate the predicting factor for the development of LV dysfunction, the patients were divided into two groups with and without LV dysfunction by defining the LV dysfunction as $\triangle \mathrm{LVEF}>-6 \%$, that is, the decrease in LVEF beyond its standard deviation. As a result, 13 patients exhibited LV dysfunction during the 4-8-year observation, and the clinical parameters were compared with those in the rest of the patients (table 2). Figure 8 shows the time course of the changes in LVEF and LVDd data in the 13 patients with LV dysfunction. Although there were some variations, most patients exhibited gradual changes in those parameters, and any critical time point for the changes in these parameters was not detected. In the univariate analysis, age, LVEF, PVC prevalence and PVC grade at the initial evaluation exhibited a significant difference. However, in the multivariate analysis, only LVEF and PVC prevalence showed a significant difference so that these factors were considered as independent predicting factors for future development of LV dysfunction. For these two factors, statistically significant receiver operating characteristics (ROC) curves could be drawn. For the LVEF, the area under the curve (AUC) was 0.702, and the most powerful cut-off point was $68 \%$ with a sensitivity of 0.692 and a specificity of $0.650(p=0.0026)$. For the PVC prevalence, the AUC was 0.724 , and the most powerful cut-off point was 31268 beats/day with a sensitivity of 0.692 and a specificity of $0.929(\mathrm{p}<0.0001)$.
Figure 3 Relationship between the premature ventricular contraction (PVC) prevalence and change in left ventricular diastolic dimension ( $\triangle \mathrm{LVDd}$ ) during the 4year or 5.6 (1.7)-year observational periods. The $\Delta \mathrm{LVDd}$ was calculated as (LVEF at the evaluation time point) - (initial LVEF). The left panel shows the data after the basic 4-year follow-up, and the right panel the data at the latest follow-up. Although most of the patients exhibited only small changes in the LVDd measurement, several patients exhibited a relatively large increase in the LVDd, and there was a weak but significant positive correlation between the PVC prevalence and $\triangle$ LVDd for both the 4-year and 5.6 (1.7)-year follow-ups. See text for details.
4 years

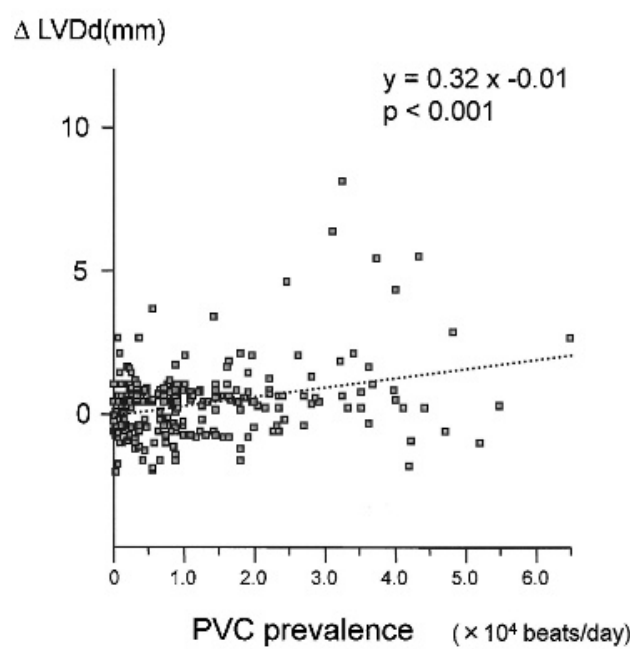

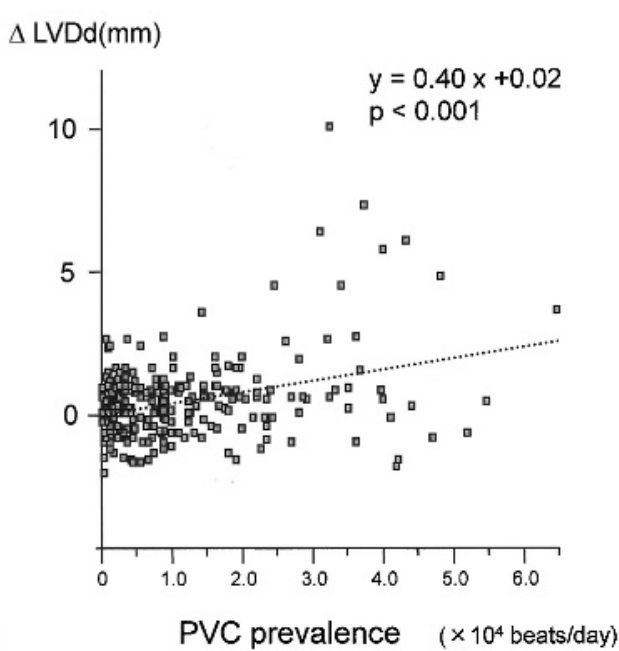


Figure 4 Relationship between the change in left ventricular ejection fraction $(\triangle \mathrm{LVEF})$ and change in left ventricular diastolic dimension ( $\triangle \mathrm{LVDd}$ ) at 4 years at the latest time point of observation. At both time points of observation, $\triangle \mathrm{LVEF}$ and $\Delta$ LVDd exhibited a strong significant correlation, indicating that these changes appeared in each patient proportionally. See text for details.

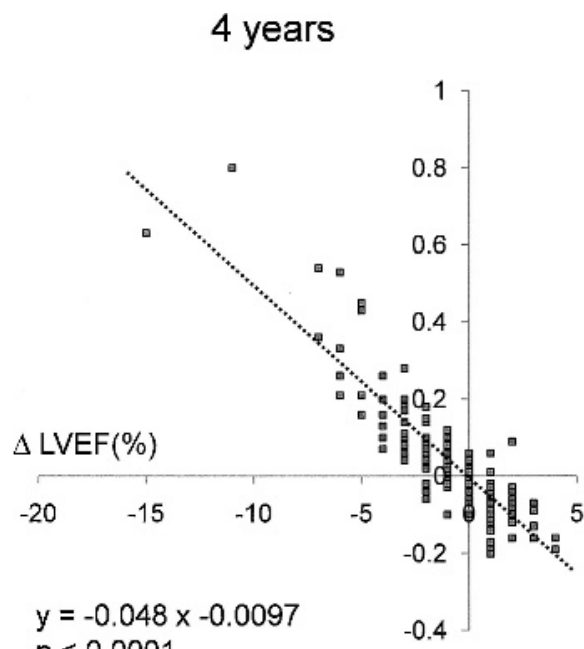

$\Delta$ LVDd mm
Latest $(5.6 \pm 1.7$ years $)$

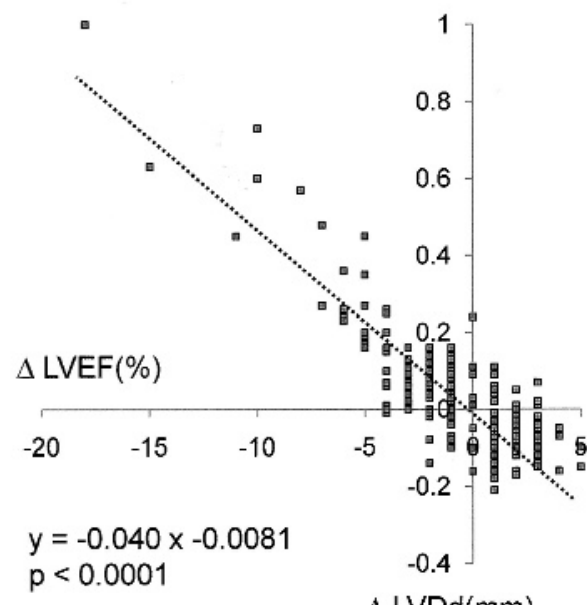

$\triangle \operatorname{LVDd}(\mathrm{mm})$

\section{DISCUSSION}

\section{Prognostic significance of frequent PVCs originating from the ventricular outflow tract}

Although the relationship between frequent PVCs originating from the ventricular outflow tract and arrhythmogenic right ventricular cardiomyopathy was once suggested, ${ }^{11}{ }^{12}$ in most patients with frequent PVCs, but without any structural heart disease, those PVCs are now considered to be a clinically benign condition. ${ }^{13}$ However, some recent studies have documented that the LV dysfunction in patients with frequent PVCs could recover after elimination of the PVCs by catheter ablation therapy. ${ }^{6-10}$ At least in those cases, the frequent PVCs were considered to be the primary mechanism causing the LV dysfunction because the LV systolic function mostly recovered after the simple elimination of the PVCs. However, the clinical importance of frequent PVCs in patients without any LV dysfunction is still unclear because, to our knowledge, no studies have documented the natural history in such patients. In the present study, a prospective cohort observation was performed in patients with frequent PVCs without any LV dysfunction, and the study documented several new important findings. First, when patients with symptoms with VT/NSVT were eliminated during the baseline evaluation, the remaining asymptomatic or less symptomatic patients did not exhibit any

serious clinical events such as syncope, new-onset VT/VF or overt heart failure during an observation period of 4 years or 5.8 (2.3) years. Second, although the mean LVEF did not exhibit any significant change during the observation period in any of the patients, there were several cases who exhibited a considerable decrease in the LVEF, and there was a weak but significant relationship between the PVC prevalence and change in the LVEF ( $\triangle \mathrm{LVEF}$ ). Third, when the patients were divided according to the PVC prevalence, the patients with highly frequent PVCs ( $>20000$ beats/day) exhibited a significant decrease in the LVEF at the 4- and 5.8 (2.3)-year follow-ups. Finally, the PVC prevalence and LVEF at the initial evaluation were considered independent predicting factors for future development of LV dysfunction. To the best of our knowledge, these findings are novel and indicated the prognostic significance of frequent PVCs even in the asymptomatic patients with a normal LV function when the PVC prevalence was high.

\section{Mechanism of the LV dysfunction caused by frequent PVCs}

The mechanism of the LV dysfunction observed in the patients with frequent PVCs was not clear. Several reports have suggested a mechanism involving tachycardia-induced cardiomyopathy as the cause of the LV dysfunction, ${ }^{8-10} 1415$ but that can be questioned because the total number of heart beats
Figure 5 Change in the left ventricular ejection fraction (LVEF) and left ventricular diastolic dimension (LVDd) over the time course in the groups with a different premature ventricular contraction (PVC) prevalence. The less frequent PVC group did not exhibit any significant change in the LVEF or LVDd, but the highly and moderately frequent PVC groups exhibited a significant decrease in the LVEF and a significant increase in the LVDd. See text for details.
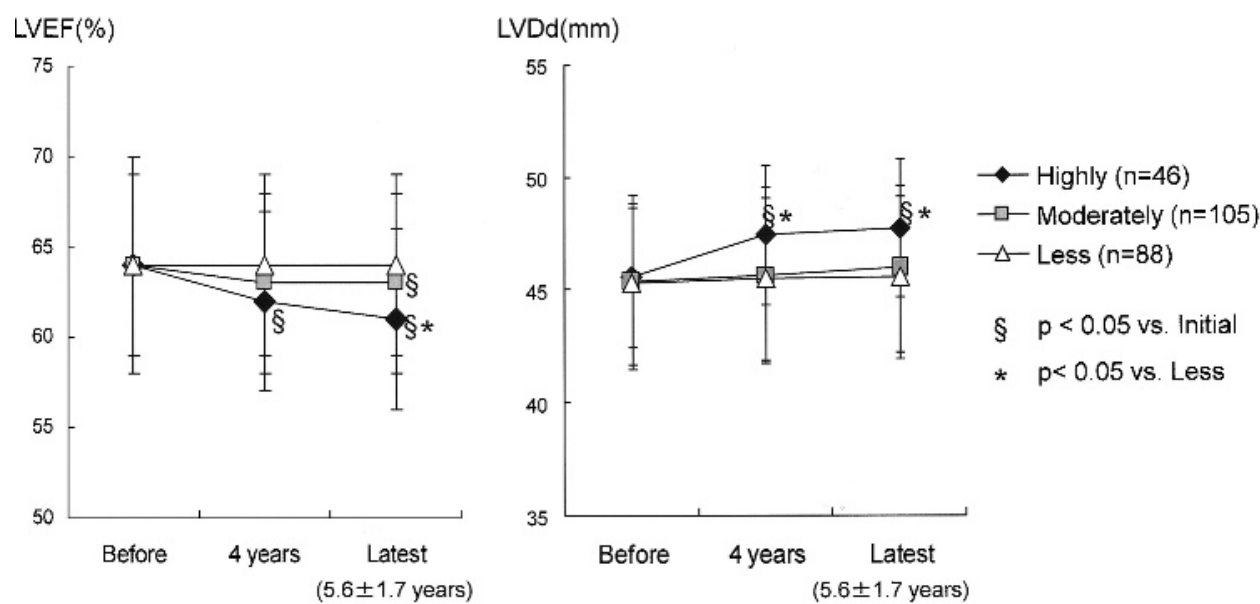
Figure 6 Comparison of the change in left ventricular ejection fraction ( $\triangle \mathrm{LVEF}$ ) (upper panels) and change in left ventricular diastolic dimension ( $\Delta \mathrm{LVDd}$ ) (lower panels) among the three groups with less, moderately and highly frequent premature ventricular contraction (PVC) prevalence. At both the 4-year and latest follow-up visits, the decrease in the LVEF, that is, negative $\triangle \mathrm{LVEF}$, and the increase in the LVDd, that is, positive $\triangle$ LVDd, were larger in the highly frequent PVC group than in the less and moderately frequent PVC groups. See text for details.
4 years
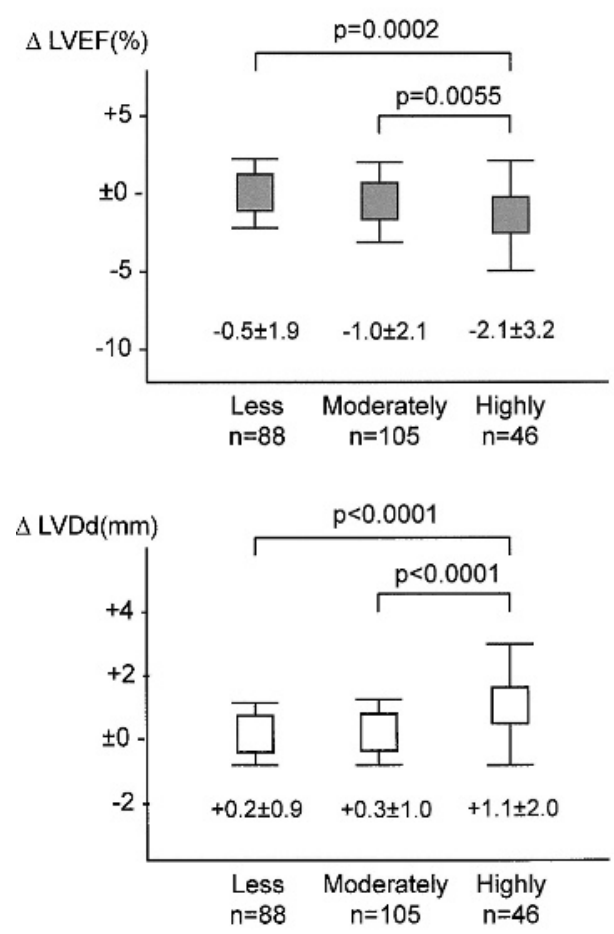

Latest $(5.6 \pm 1.7$ years $)$

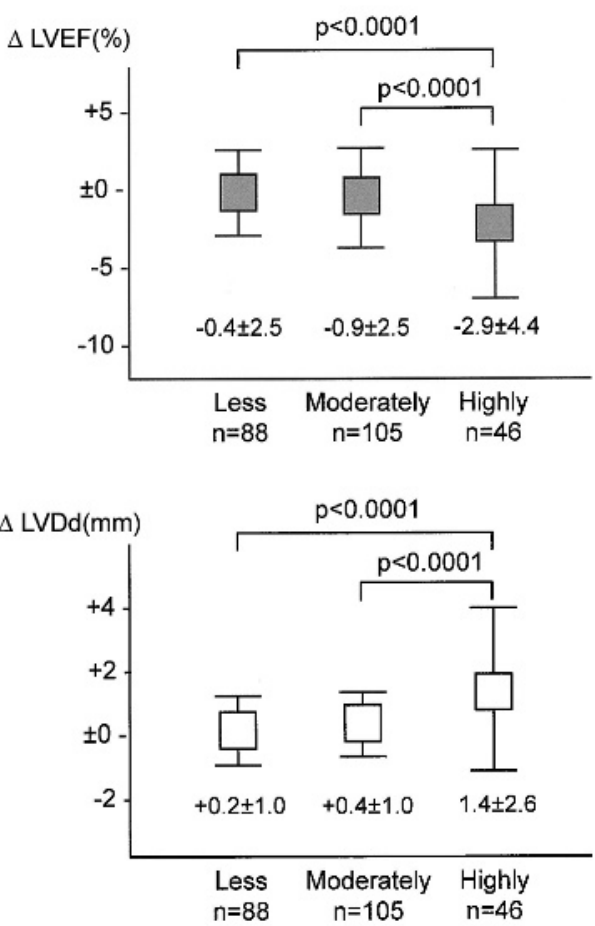

during Holter monitoring in patients with frequent PVCs would be expected to be at a level similar to that for normal patients. In contrast, a similar mechanism as bradycardia-induced LV dysfunction might be a possible mechanism ${ }^{16}{ }^{17}$ because each PVC could not produce an effective cardiac output due to the premature excitation resulting in a much lower pulse rate than in normal subjects. For example, in a patient with 30000 $40000 \mathrm{PVCs} /$ day, that patient might lose as much as almost a third of the effective cardiac output daily and might develop a similar condition as severe bradycardia, such as sick sinus syndrome or atrioventricular block.

The possibility of the existence of occult structural heart disease could not be completely denied. ${ }^{8-10} 18$ Although structural heart disease was carefully ruled out by using echocardiography and cardiac magnetic resonance imaging in the present study, the existence and progression of a subnormal or slightly abnormal condition could not be detected during the baseline evaluation when the parameters were within normal limits. In fact, the change in the LVEF during the observation period varied among the patients. Even in the patient group with highly frequent PVCs, the LVEF did not decrease equally, and more than half of the patients did not exhibit any obvious decrease in the LVEF. This indicates that the patients who exhibited an overt decrease in the LVEF may have had some specific difference in their cardiac structure from the others, but annual evaluations utilising echocardiography or Holter ECG recordings could not detect any structural abnormalities, even in such patients with a larger $\triangle \mathrm{LVEF}$. Invasive examinations such as cardiac catheterisation, coronary angiography or myocardial biopsy may reveal some abnormalities, but they
Figure 7 Relationship between the follow-up period and change in left ventricular ejection fraction ( $\Delta$ LVEF) and left ventricular diastolic dimension $(\Delta \mathrm{LVDd})$. This figure shows the relationship between the observation period and the $\triangle \mathrm{LVEF}$ (left panel) and $\Delta$ LVDd (right panel) in all patients. In the patients who exhibited a larger decrease in the LVEF or increase in the LVDd, the change seemed to appear within a relatively early period, that is, 4-8 years. There was no significant correlation between the follow-up period and the $\Delta \mathrm{LVEF}$ or $\Delta \mathrm{LVDd}$. See text for details.
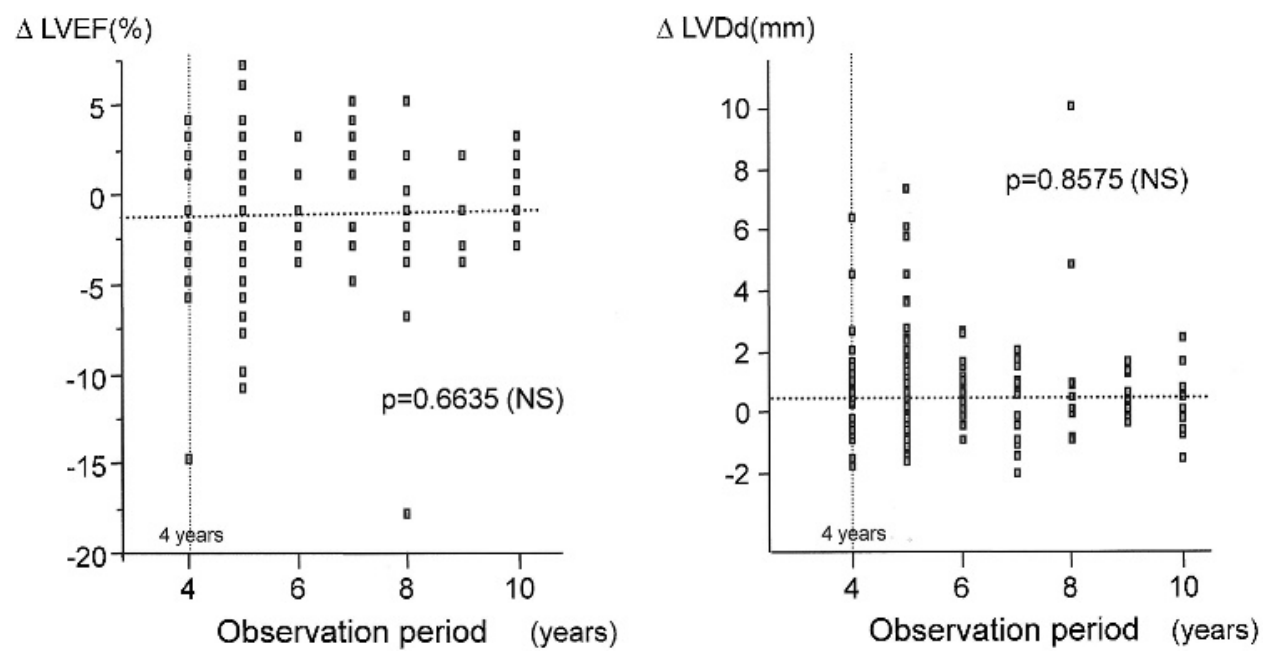
Figure 8 Time course of changes in left ventricular ejection fraction (LVEF) and left ventricular diastolic dimension (LVDd) in 13 patients with the development of left ventricular dysfunction. Although there were some variations, most of the patients exhibited gradual changes in these parameters. See text for details.

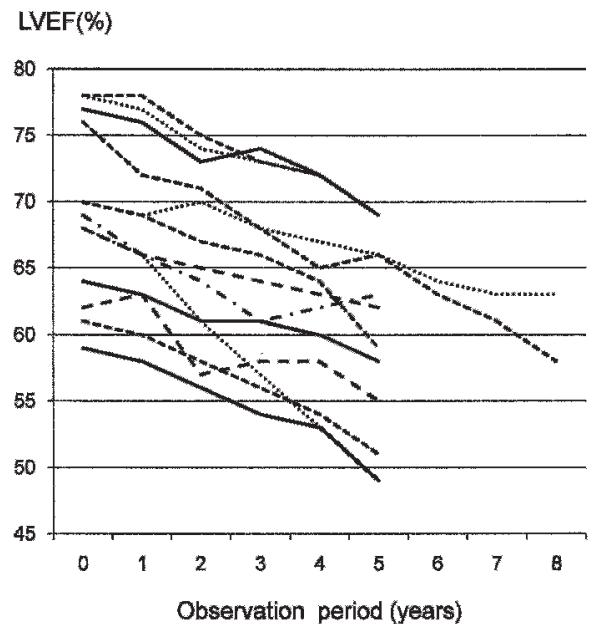

could not be ethically indicated in the present study because no patients exhibited any clinical problems.

\section{Determinants of the appearance of LV dysfunction}

In the present study, it was documented that the PVC prevalence was one of the determinants of the appearance of LV dysfunction during the long-term follow-up. That was compatible with the idea of bradycardia-induced LV dysfunction because more frequent PVCs would result in a more frequent loss of an effective cardiac output. ${ }^{16-18}$ The most recent report by Bogun et al documented the negative correlation between the LVEF and PVC prevalence at the time of catheter ablation therapy, ${ }^{9}$ and that also indirectly indicated that the PVC prevalence was an important determinant of the change in the LVEF. Another important issue was that a higher LVEF at the initial evaluation was independently correlated with the development of LV dysfunction. The mechanism of this result was unclear but higher contractility may lead to larger oxygen consumption, which then may lead to an earlier appearance of LV dysfunction at least in this mechanism of high PVC prevalence. Although the contraction period of PVCs was thought to be another determinant of LV dysfunction, there was no significant relationship observed between the observation period and $\triangle \mathrm{LVEF}$ in the present study. That result might have been influenced by the limitation of the determination of when the period of experiencing PVCs began. Because the observation period was started only after the patient underwent a consultation at the outpatient clinic, the observation period in this study might not have reflected the real initiation period of the PVC burden. However, because the LVEF data in this study were evaluated by the $\triangle$ LVEF during the observation period, the change in the LVEF was considered to reflect the 4- or 5.8 (2.3)year observation, but not the long-term preobservation period prior to the enrolment of the patients in the study. The results of this study clearly indicate that at least there might have been some patients who would have exhibited an obvious decrease in

Table 2 Uni- and multivariate analysis of comparison of patients with and without the appearance of left ventricular (LV) dysfunction

\begin{tabular}{|c|c|c|c|c|c|c|}
\hline & \multirow{2}{*}{$\begin{array}{l}\text { With LV } \\
\text { dysfunction }\end{array}$} & \multirow{2}{*}{$\begin{array}{l}\text { Without LV } \\
\text { dysfunction }\end{array}$} & \multirow{2}{*}{$\begin{array}{l}\text { Univariate } \\
\text { p Value }\end{array}$} & \multicolumn{2}{|l|}{ Multivariate } & \multirow[b]{2}{*}{ p Value } \\
\hline & & & & $95 \% \mathrm{CI}$ & Odds ratio & \\
\hline Number & 13 & 226 & & & & \\
\hline Age (years) & $51(12)$ & $42(12)$ & $0.013^{*}$ & -0.0964 to 0.0043 & 1.28 & 0.075 \\
\hline Gender (F:M) & $8: 5$ & $113: 113$ & 0.418 & & & \\
\hline $\begin{array}{l}\text { Systolic blood pressure } \\
(\mathrm{mm} \mathrm{Hg})\end{array}$ & $133(11)$ & $132(11)$ & 0.819 & & & \\
\hline $\begin{array}{l}\text { Diastolic blood pressure } \\
(\mathrm{mm} \mathrm{Hg})\end{array}$ & $78(8)$ & $76(7)$ & 0.265 & & & \\
\hline \multirow[t]{2}{*}{ Complications } & HT 0, HL 1 & HT 16, HL 10 & 0.311 & & & \\
\hline & DM 0 & DM 9 & & & & \\
\hline Beta-blockers (n) & 1 & 20 & 0.886 & & & \\
\hline $\begin{array}{l}\text { Left ventricular ejection } \\
\text { fraction (\%) initial }\end{array}$ & $69(7)$ & $64(6)$ & $0.004^{*}$ & $\begin{array}{l}-0.3032 \text { to } \\
-0.0572\end{array}$ & 18.2 & $0.002^{*}$ \\
\hline $\begin{array}{l}\text { Left ventricular diastolic } \\
\text { dimension }(\mathrm{mm}) \text { initial }\end{array}$ & $45(5)$ & $46(4)$ & 0.579 & & & \\
\hline PVCs (beats/day) initial & 29224 (20 181) & $11313(10916)$ & $<0.001^{*}$ & $\begin{array}{l}-0.0001 \text { to } \\
-0.0052\end{array}$ & 85.4 & $<0.001^{*}$ \\
\hline $\begin{array}{l}\text { PVC grade (highly/ } \\
\text { moderate/less) }\end{array}$ & $9 / 1 / 3$ & $37 / 104 / 85$ & $<0.001^{*}$ & -0.7182 to 1.6045 & 2.53 & 0.226 \\
\hline $\begin{array}{l}\text { NSVT (>3 beats, \%) } \\
\text { initial }\end{array}$ & 38.4 & 17.7 & 0.062 & & & \\
\hline
\end{tabular}


the LVEF even during a limited observation period. Additionally, the patients who exhibited a significant decrease in LV function may have a different genetic background because they showed different responses from the other patients, even they were in the very similar conditions. We cannot comment on this point because no genetic examination was involved in this study, but the additional examinations should be performed in future if possible.

\section{Clinical implications}

The results of this study have raised an extremely important issue concerning the clinical management of frequent PVCs. First, a benign prognosis in patients with frequent PVCs without any structural heart disease was confirmed by the 5.8 (2.3)-year prospective observation when patients with symptoms with VT/VF or heart failure were excluded at the initial evaluation. Second, although the prognosis in asymptomatic or less symptomatic patients was benign, a considerable decrease in the LVEF should be expected at least in some populations of patients with highly frequent PVCs during a 4-year follow-up or longer. The latter indicates the necessity of repeated evaluations of the LV function over the years in patients with highly frequent PVCs even without any structural heart disease at the initial evaluation. According to the results of this study, one might consider that catheter ablation therapy would be indicated in all patients with highly frequent PVCs, ${ }^{19-21}$ and that might be a fair assumption when the ablation procedure could be performed with adequate safety and accuracy. However, because the decrease in the LVEF appeared very slowly, even in the patients with a considerable $\triangle$ LVEF in our observation, we recommend that the patients first be observed over several years, and then when the LVEF exhibits a considerable decrease, catheter ablation may be indicated.

\section{Limitations}

This study included a few methodological limitations. First, there might have been a problem with the reproducibility of the data of the PVC number in the Holter monitoring and measurement of the LVEF in the echocardiogram. Even in the same patient, the PVC number may exhibit a day-to-day variation and may vary up to $5-40 \%$, and thus the evaluation of the PVC prevalence using only one Holter recording could possibly be misleading for the real PVC prevalence in each case. In the present study, we employed the PVC prevalence as the mean PVC number from two or three Holter recordings in order to exclude any day-to-day variation. As a result, the PVC prevalence did not exhibit any large changes even during the reevaluation of the Holter ECGs every year in each case. For the evaluation of the LVEF, we also employed the mean LVEF from plural recordings for the LVEF representing each time point. Second, the number of patients and observation period were limited. To precisely discuss the long-term prognosis, a longer observation period with a larger number of patients would be necessary. Third, we did not perform any invasive evaluations such as cardiac catheterisation, coronary angiography or myocardial biopsy even in the patients with a considerable $\triangle$ LVEF during the observation period. This was simply due to ethical reasons because no patients exhibited any active clinical symptoms such as heart failure. Such invasive evaluations may have revealed some underlying structural changes especially in the patients with a larger $\triangle$ LVEF, but we ethically decided that those evaluations should be indicated only after further observation.

\section{CONCLUSIONS}

The prognosis in the patients with frequent PVCs without any structural heart disease appeared benign after at least a 4-year follow-up when the patients with symptoms due to VT/VF or LV dysfunction were excluded at the initial evaluation. However, because the patients with highly frequent PVCs may exhibit a considerable decrease in the LVEF, the possibility of LV dysfunction should be given attention during the longterm follow-up.

Funding: This study was partly supported by a Grant in Aid for Scientific Research 2006 from the Ministry of Education and Science.

Competing interests: None

Ethics approval: Ethics approval was provided by the Clinical Studies and Ethics Committee of Kitasato University Hospital.

Patient consent: Obtained.

\section{REFERENCES}

1. Gaita $\mathbf{F}$, Giustetto $C$, Di Donnna P, et al. Long-tern follow-up of right ventricular monomorphic extrasystoles. J Am Coll Cardiol 2001;38:364-70.

2. Vijgen J, Hill P, Biblo LA, et al. Tachycardia-induced cardiomyopathy secondary to right ventricular outflow tract ventricular tachycardia: improvement of left ventricular systolic function after radiofrequency catheter ablation of the arrhythmia. J Cardiovasc Electrophysiol 1997;8:445-50.

3. Chugh SS, Shen WK, Luria DM, et al. First evidence of premature ventricular complex-induced cardiomyopathy: a potentially reversible cause of heart failure. $J$ Cardiovasc Electrophysiol 2000;11:328-9.

4. Grimm W, Menz V, Hoffmann J, et al. Reversal of tachycardia induced cardiomyopathy following ablation of repetitive monomorphic right ventricular outflow tract tachycardia. Pacing Clin Electrophysiol 2001;24:166-71.

5. Shiraishi $\mathbf{H}$, Ishibashi $\mathrm{K}$, Urao $\mathrm{N}$, et al. A case of cardiomyopathy induced by premature ventricular complexes. Circ $J$ 2002;66:1065-7.

6. Zalzstein $\mathbf{E}$, Wagshal A, Zucker N, et al. Ablation therapy of tachycardia-related cardiomyopathy. Isr Med Assoc J 2003;5:64-5

7. Redfearn DP, Hill JD, Keal R, et al. Left ventricular dysfunction resulting from frequent unifocal ventricular ectopics with resolution following radiofrequency ablation. Europace 2003;5:247-50.

8. Takemoto M, Yoshimura H, Ohba Y, et al. Radiofrequency catheter ablation of premature ventricular complexes from right ventricular outflow tract improves left ventricular dilation and clinical status in patients without structural heart disease. J Am Coll Cardiol 2005;45:1259-65.

9. Bogun F, Crawford T, Reich S, et al. Radiofrequency ablation of frequent, idiopathic premature ventricular complexes: Comparison with a control group without intervention. Heart Rhythm 2007;4:863-7.

10. Sekiguchi Y, Aonuma K, Yamauchi Y, et al. Chronic hemodynamic effects after radiofrequency catheter ablation of frequent monomorphic ventricular premature beats. J Cardiovasc Electrophysiol 2005;16:1057-63.

11. O'Donnell D, Cox D, Bourke J, et al. Clinical and electrophysiological differences between patients with arrhythmogenic right ventricular dysplasia and right ventricular outflow tract tachycardia. Eur Heart J 2003;24:801-10.

12. Jaoude SA, Leclercq JF, Coumel P. Progressive ECG changes in arrhythmogenic right ventricular disease. Evidence for an evolving disease. Eur Heart J 1996;17:1717-22.

13. Wakisaka Y, Niwano S, Sasaki T, et al. Evaluation of long-term prognosis of ventricular arrhythmias originating from right ventricular outflow tract (RVOT) [abstract]. Circulation 2004:110:III-694-5.

14. Yarlagadda RK, Iwai S, Stein KM, et al. Reversal of cardiomyopathy in patients with repetitive monomorphic ventricular ectopy originating from the right ventricular outflow tract. Circulation 2005;112:1092-7.

15. Goyal R, Harvey M, Daoud EG, et al. Effect of coupling interval and pacing cycle length on morphology of paced ventricular complexes. Implications for pace mapping. Circulation 1996;94:2843-9.

16. Kennedy HI, Pescarmona JE, Bouchard RJ, et al. Objective evidence of occult myocardial dysfunction in patients with frequent ventricular ectopy without clinically apparent heart disease. Am Heart J 1982;104:57-65.

17. Hoffman BF, Bartelstone HJ, Scherlag BJ, et al. Effects of postextrasystolic potentiation on normal and failing hearts. Bull NY Acad Med 1965;41:498-534.

18. Lemery R, Brugada P, Bella PO, et al. Nonischemic ventricular tachycardia. Clinical course and long-term follow-up in patients without clinically overt heart disease. Circulation 1989;79:990-9.

19. Duffee OF, Shen WK, Smith HC. Suppression of frequent premature ventricular contractions and improvement of left ventricular function in patients with presumed idiopathic dilated cardiomyopathy. Mayo Clin Proc 1998;73:430-3.

20. Wellens HI. Radiofrequency catheter ablation of benign ventricular ectopic beats: a therapy in search of a disease? J Am Coll Cardiol 1995;26:850-1.

21. Zhu OW, Maloney J0, Simmons TW, et al. Radiofrequency catheter ablation for management of symptomatic ventricular ectopic activity. J Am Coll Cardiol 1995:26:843-9. 